Distribution Category:

Mathematics and Computers (UC-32)

\title{
ANI-B2-4
}

ARGONNE NATIONAL LABORA'TORY

9700 South Cass Avenue

Argonne, Illinois 60439

MNIS $--82-4$

DE8 2008087

\section{IMPROVING THE ACCURACY OF COMPUTED SINGULAR VALUES}

\section{J.J. Dongarra}

Applied Mathematics Division

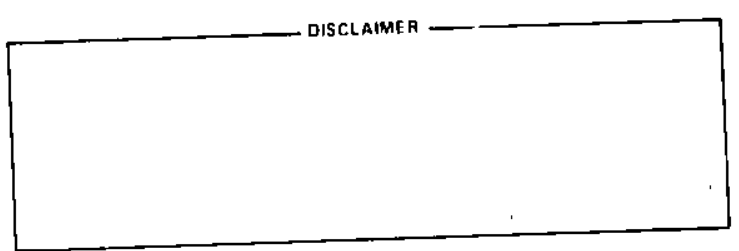

January 1982 


\begin{abstract}
This paper describes a computational method for impruving the accuracy of a given singular value and its associated left and right singular vectors. The method is analogous to iterative improvement for the solution of linear systems. That is, by means of a low-precision computation, an iterative algorithm is applied to increase the accuracy of the singular value and vectors; extended precision computations are used in the residual calculation. The method is related to Newton's Method applied to the singular value problem and inverse iteration for the eigenvalue problem.
\end{abstract}




\title{
Improving the Accuracy of Computed Singular Values
}

\author{
J.J. Dongarra * \\ Argonne National Laboratory
}

\begin{abstract}
Simmary - This paper describes a computational method for improving the accuracy of a given singular value and its associated left and right singular vectors. The method is analogous to iterative improvement fo: the solution. of linear systems. That is, by means of a low-precision computation, an iterative algorithm is applied to increase the accuracy of the singular value and vectors; extended precision compulations are used in the residual calculation. The method is related to Newton's Method applied to the singular value problem and inverse iteration for the eigenvalue problem.
\end{abstract}

\section{THE BASIC ALGORITHM}

In a recent paper. Dongarra, Moler and Wilkinson [1] described an algorithm for improving an approximation to a simple eigenvalue and the corresponding eigenvector. In this paper we extend and medify the algoriltm to cover the singular value problem.

We begin with a bricf description of the basic algorithm.

Given an $m \times n$ rectangular matrix $A$, we are interested in the decomposition

$$
A=U \Sigma V^{T}
$$

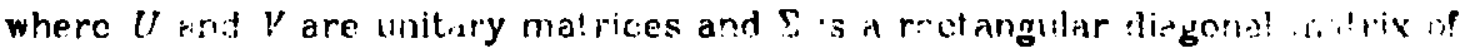
the same dimension us $A$ with real nonnegative dingonal entries. The equations can also be written as

\footnotetext{
- Work supported in part by the Applied Matheriatical Sciencen Resenrch Program (Kr-04-02)

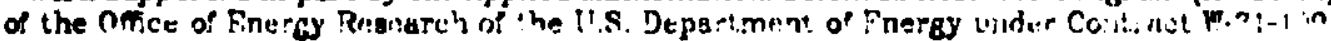
Rng-sy.
} 


$$
A u_{i}=\sigma_{i} u_{i}
$$

and

$$
A^{T} u_{i}=\sigma_{i} \nu_{i} \text { for each singular value } \sigma_{i}
$$

If $\sigma, u$, and $v$ have been derived from some computation on a computer with finite precision or by some insight into the problem, they are generally not the true singular value and vectors, but approximations. We know, however, that there exist $\mu_{1}, \mu_{2}, y$, and $z$ such that

$$
A(v+y)=\left(\sigma+\mu_{1}\right)(u+z)
$$

and

$$
A^{T}(u+z)=\left(\sigma+\mu_{2}\right)(v+y)
$$

where $\mu_{1}, \mu_{2}, y$, and $z$, when added to computed $\sigma, u$, and $v$, give the exact left and right singular vectors and the exact singular value. The corrections $\mu_{1}$ and $\mu_{e}$ come about by the separate na:ure of Equations $1 . P$ and 1.3. We compute the correction to $\sigma$ as $\mu=\left(\mu_{1}+\mu_{2}\right) / 2$.

The above equations can be expanded to obtain

$$
A y-\sigma z-\mu_{1} u=\sigma u-A v+\mu_{1} z
$$

and

$$
A^{T} z-\sigma y-\mu_{2} v=\sigma v-A^{T} u+\mu_{2} y
$$

If the orthogonality conditicns

$$
(v+y)^{r}(v+y)=1
$$

and

$$
(u+z)^{T}(u+z)=1
$$

are included, we then have $m+n+2$ equations in $m+n+2$ unkrowns. We can now rewrite the equations in matrix notations to obtain 


$$
\left(\begin{array}{cccc}
-\sigma I & A & -u & 0 \\
A^{T} & -\sigma I & 0 & -v \\
2 u^{T} & 0 & 0 & 0 \\
0 & 2 v^{T} & 0 & 0
\end{array}\right)\left(\begin{array}{l}
z \\
y \\
\mu_{1} \\
\mu_{2}
\end{array}\right)=\left(\begin{array}{c}
\sigma u-A v+\mu_{1} z \\
\sigma v-A^{T} u+\mu_{2} y \\
1-u^{T} u-z^{T} z \\
1-v^{T} v-y^{T} y
\end{array}\right)
$$

Note that this is a mildly nonlinear matrix equation. We can determine the unknowns $\left(z, y, \mu_{1}, \mu_{2}\right)^{T}$ iteratively by solving

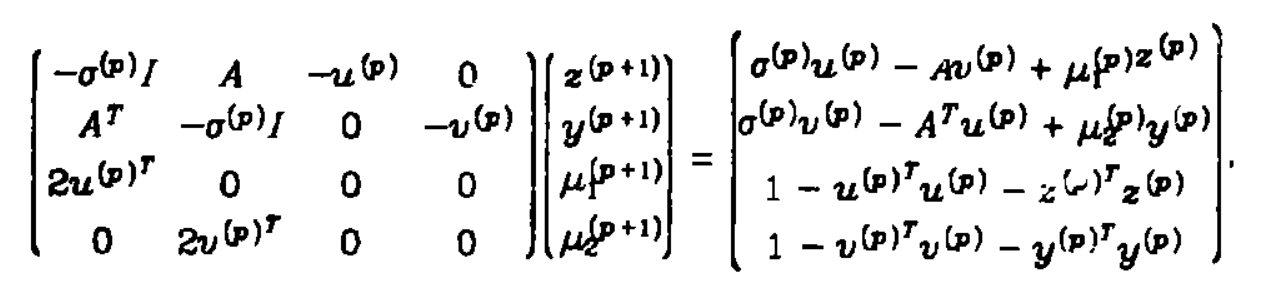

to obtain corrections to $u^{(p)}, v^{(p)}$, and $\mu^{(p)}$ by the updates

$$
\begin{aligned}
& u^{(p+1)}=u^{(p)}+z^{(p+1)} \\
& v^{(p+1)}=v^{(p)}+y^{(p+1)} \\
& \sigma^{(p+1)}=\sigma^{(p)}+\left(\mu p^{(p+1)}+\mu^{(p+1)}\right) / 2 .
\end{aligned}
$$

If $A$ is $m \times n$, then this is an $(m+n+2) \times(m+n+2)$ system to be solved. If this system is solved, we can compute corrections $\mu, y$, and $z$ to the singular value and the singular vectors, thereby obtaining a more accurate value for the singular value and singular vectors.

If we handle this as we do in the eigenvalue case[1], we will improve the accuracy of $\sigma, u$, and $v$. The accuracy obtained by the algorithm will be full working precision, with only the residual calculations (the right-hand side of Equation 1.9) done in extended precision.

\section{RMAMTONSHIP TO NEWTON'S YBTHOD}

The algorithm as described above can be derived by the use of Newion's Mothod applied to Equations (1.2) and (1.3). We defne functions $f_{1}$ and $f$ as fol- 
lows:

$$
\begin{aligned}
& f_{1}\left(u, v, \sigma_{1}, \sigma_{2}\right)=A v-\sigma_{1} u \\
& f_{2}\left(u, v, \sigma_{1}, \sigma_{2}\right)=A^{T} u-\sigma_{2} v \\
& f_{s}\left(u, v, \sigma_{1}, \sigma_{2}\right)=u^{T} u-1 \\
& f_{4}\left(u, v, \sigma_{1}, \sigma_{2}\right)=v^{T} v-1
\end{aligned}
$$

and

$$
f(x)=\left(f_{1}(x), f_{2}(x), f_{3}(x), f_{4}(x)\right)
$$

where

$$
x=\left(\begin{array}{l}
u \\
v \\
\sigma_{1} \\
\sigma_{2}
\end{array}\right)
$$

The approach is to find the zeros of $f(x)$. Newton's Method applied to this problem is

$$
f^{\prime}\left(x_{i}\right)\left(x_{i+1}-x_{i}\right)=-f\left(x_{i}\right) .
$$

where

$$
x_{i}=\left(\begin{array}{c}
u^{(i)} \\
v^{(i)} \\
\sigma_{f^{(i)}}^{(i)} \\
\sigma_{2}^{(i)}
\end{array}\right)
$$

The derivative of $f(x)$ is

$$
f^{\prime}(x)=\left(\begin{array}{cccc}
-\sigma_{1} I & A & -u & 0 \\
A^{T} & -\sigma_{2} I & 0 & -v \\
2 u^{T} & 0 & 0 & 0 \\
0 & 2 v^{T} & 0 & 0
\end{array}\right)
$$

The above method expressed in matrix notation is then just a restatement of Equation (1.8), ignoring the second order terms in the right hand side.

Notice that since the method is equivalent to Newton's Method, we could compute tbe left and right singular vectors, given a close approximation to tho oingular value. 


\section{EFPECTS OP VARIOUS FACTORJZATIONS}

If we have computed the singular value decomposition and retained the matrices produced during the factorization, each singular value and the corresponding singular vectors can be improved in $O(m n)$ operations. We will assume that the matrices $U, \Sigma$, and $V$ are available such that $A \approx U \Sigma V^{T}$. Then the coefficient matrix in Equation 1.8 can be decomposed into the form

$$
\left(\begin{array}{cccc}
U & 0 & 0 & 0 \\
0 & V & 0 & 0 \\
0 & 0 & 1 & 0 \\
0 & 0 & 0 & 1
\end{array}\right)\left(\begin{array}{cccc}
-\sigma_{8} I & \Sigma & -\varepsilon_{*} & 0 \\
\Sigma & -\sigma_{8} I & 0 & -\varepsilon_{z} \\
\theta_{E}^{T} & 0 & 0 & 0 \\
0 & e_{*}^{T} & 0 & 0
\end{array}\right)\left(\begin{array}{cccc}
U^{T} & 0 & 0 & 0 \\
0 & V T & 0 & 0 \\
0 & 0 & 1 & 0 \\
0 & 0 & 0 & 1
\end{array}\right)
$$

where $e_{*}$ is the $s^{\text {th }}$ column of the identity matrix, and $\sigma_{*}$ is the approximation being improved.

This factored form can be used to simplify Equation (1.8). Since $U$ and $V$ are orthogonal, systems of equations involving the left and the right matrices of (3.1) can be easily solved by simply multiplying by the transposes. Systems of equations involving the matrix in the center can be handled by solving $2 \times 2$ or $4 \times 4$ subsystems of equations as can be seen from the non-zero structure of the matrix:

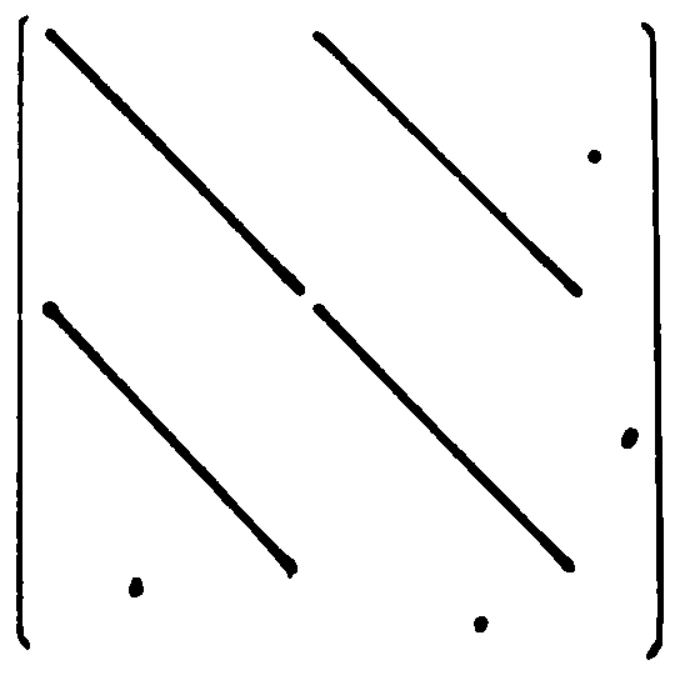


If we have a bidiagonal factorization of $A$, say $A=U B V^{T}$, where $B$ is bidiagonal, then we can improve the accuracy in $O(m n)$ operations. Let us assume we have the matrices $V$ and $B$ from the bidiagonalization procedure. We will concentrate only on the matrix

$$
\left(\begin{array}{cc}
-\sigma I & A \\
A^{T} & -\sigma I
\end{array}\right)
$$

This matrix is the interesting part of the one in (1.8) and can be thought of as a rank 2 modification of that equation. The matrix can then be written as

$$
\left(\begin{array}{cc}
I & 0 \\
-\frac{1}{\sigma} A^{T} & V T
\end{array}\right)\left(\begin{array}{cc}
-\sigma I & 0 \\
0 & -\sigma I+\frac{1}{\sigma} B^{T} B
\end{array}\right)\left(\begin{array}{cc}
I & -\frac{1}{\sigma} A \\
0 & V
\end{array}\right) .
$$

Note that solving systems based on this factored form is a simple task since $V^{-1}=V^{r}$. The only actual need for an equation solver comes from

$$
-\sigma I+\frac{1}{\sigma} B^{T} B
$$

and this matrix is tridiagonal. Thus, given the bidiagonal matrix and the $V$ matrix of the transformation, we can improve the accuracy of the singular values.

If we have instead the $Q R$ factorization of $A$, namely $A=Q R$, where $R$ is upper triungulor and $Q$ is orthogonal, then we can improve the accuracy of the singular value in $O\left(m n+n^{3}\right)$ operations, provided we have some approximation to it. We will concentrate on the matrix in (3.3). This matrix can be rewritten in factored form as

$$
\left(\begin{array}{cc}
I & 0 \\
-\frac{1}{\sigma} A^{r} & Q^{r}
\end{array}\right)\left(\begin{array}{cc}
-\sigma I & 0 \\
0 & -\sigma I+\frac{1}{\sigma} R^{r} R
\end{array}\right)\left(\begin{array}{cc}
I & -\frac{1}{\sigma} A \\
0 & Q
\end{array}\right) .
$$


As in (3.4) it becomes a matter of solving equations with a matrix of the form

$$
-\sigma I+\frac{1}{\sigma} R^{T} R
$$

Unlike Equation (3.5), this matrix is full and, unfortunately, the factor $R$ cannot be used to simplify the process since the matrix $R^{T} R$ is being modified by a rank $n$ matrix, $\sigma I$. Equation (3.7) requires a further factorization to solve systems based upon it.

\section{CONVERGLNCE OP THE UPDATE PROCESS}

The convergence results for this method are the same as for the eigenvalue case. We state the results here but omit the proof which can be found in [1].

In the presence of round-off error, if the initial error in the singular value is small enough in some sense and the singular value is an isolated one, the iterative process uill converge.

If working precision is used in computing the approximate singular values and extended precision is used in the residual caiculation, then when the mothod converges, if produces results that are accurale to at least full working precision.

The method is equivalent to Newton's method; therefore, the convergence is quadratic.

The method just described has a deficiency: When there are multiple singular values, the matrix in Equation (1.8) becomes ill-conditioned. The degrec of lll-conditioning is related to the separation between the singular value being improved and its closest neighbor. For identical singular values, the matrix involved is exactly singular. 
This deficiency can be illustrated by an example. For a $2 \times 2$ system the matrix has the form

$$
\left(\begin{array}{cc}
-\sigma & \sigma_{i} \\
\sigma_{i} & -\sigma
\end{array}\right)
$$

where $\sigma$ is an approximation to $\sigma_{a}$. If any $\sigma_{i}$ is close to $\sigma_{a}$, then this system will be ill-conditioned, and the conditioning depends upon $\frac{1}{\sigma-\sigma_{i}}$. In this situation one cannot improve just one singular value but must work with a cluster of them, as well as the invariant subspace of singular vectors.

\section{RESULTS}

The following numerical tests were run on a VAX 11/7BO. The initial reduction was performed in single precision; double precision was used only to compute the residuals and to add the correction to the previous result. In single precision, the working accuracy is $2^{-2}$; in double precision, the accuracy is $2^{-50}$.

The matrices used here come from the original paper by Golub and Reinsch [2]. The first matrix has the form

$$
A=\left(\begin{array}{rrrrr}
22 & 10 & 2 & 3 & 7 \\
14 & 7 & 10 & 0 & 8 \\
-1 & 13 & -1 & -11 & 3 \\
-3 & -2 & 13 & -2 & 4 \\
9 & 8 & 1 & -2 & 4 \\
0 & 1 & -7 & 5 & -1 \\
2 & -6 & 6 & 5 & 1 \\
4 & 5 & 0 & -2 & 2
\end{array}\right)
$$

with singular values

$$
\sigma_{1}=\sqrt{1248,}, \sigma_{2}=20, \sigma_{3}=\sqrt{384}, \sigma_{4}=\sigma_{0}=0
$$

The results from the improvement algorithm on this problem are given below. All results were achieved using single precision computations except to accumulate the residuals. The method used was based on the factored form of Fquation 
(3.1).

\begin{tabular}{|c|c|c|c|}
\hline Iteration & $\sigma$ & $u^{T} \underline{z i}$ & $y^{T} v$ \\
\hline $\begin{array}{c}0 \\
1 \\
2 \\
\text { true }\end{array}$ & $\begin{array}{l}35.3270149 \\
35.327043465315658 \\
35.327043465311387 \\
35.327043465311387419\end{array}$ & $\begin{array}{l}0.999999718 \\
1.000000000000101 \\
1.000000000000000\end{array}$ & $\begin{array}{l}0.999999683 \\
1.00 C 500000000304 \\
1.000000000000000\end{array}$ \\
\hline $\begin{array}{c}0 \\
1 \\
2 \\
\text { true }\end{array}$ & $\begin{array}{l}19.9999790 \\
20.000000000006048 \\
20.000000000000000 \\
20 .\end{array}$ & $\begin{array}{l}0.999999520 \\
1.000000000003621 \\
1.000000000000000\end{array}$ & $\begin{array}{l}0.999999326 \\
1.000000000003431 \\
1.000000000000000\end{array}$ \\
\hline $\begin{array}{c}0 \\
1 \\
2 \\
\text { true }\end{array}$ & $\begin{array}{l}19.5958881 \\
19.59591794227 ? 176 \\
19.595917942265425 \\
19.595917942265424785\end{array}$ & $\begin{array}{l}0.999999043 \\
1.0000<7000003258 \\
1.000000000000000\end{array}$ & $\begin{array}{l}0.999999379 \\
1.000000000003183 \\
1.000000000000000\end{array}$ \\
\hline $\begin{array}{c}0 \\
1 \\
2 \\
\text { true }\end{array}$ & $\begin{array}{l}0.00000718535284 \\
-0.000000000004162 \\
0.000000000000000 \\
0.0\end{array}$ & $\begin{array}{l}0.999998454 \\
1.000000000000745 \\
1.000000000533098\end{array}$ & $\begin{array}{l}0.699999228 \\
1.000000000000306 \\
1.000000000281307\end{array}$ \\
\hline $\begin{array}{c}0 \\
1 \\
2 \\
\text { true }\end{array}$ & $\begin{array}{l}0.00000120505399 \\
-0.000000000000479 \\
0.000000000000000 \\
0.0\end{array}$ & $\begin{array}{l}0.999998900 \\
1.000000000000304 \\
1.000000018476308\end{array}$ & $\begin{array}{l}0.999999509 \\
1.000000000000061 \\
1.000000001164373\end{array}$ \\
\hline
\end{tabular}

The results here show the iteration converging very rapidly. The singular values are initially correct to working precision, and two iterations have gained full extended precision.

For the next example we use a standard symmetric eigenvalue problem. The matrix, $w_{2 k+1}^{+}[3]$, is symmetric tridiagonal, and has some pathologically close eigenvalues and singular values. It is defined by the relations

$$
\begin{aligned}
& a_{i}=k+1-i, \quad i=1, \cdots, k+1 \\
& a_{i}=i-k-1, \quad i=k+2, \cdots, 2 k+1 \\
& \beta_{i}=1, \quad i=2, \cdots, 2 k+1
\end{aligned}
$$

where $k=5, \alpha_{i}$ is the $i^{\text {th }}$ diagonal element, and $\beta_{i}$ is the $i^{\text {th }}$ subdiagonal element. 


\begin{tabular}{|c|c|c|c|}
\hline Iteration & $\sigma$ & $u^{T} u$ & $v^{T} v$ \\
\hline 0 & 5.7462210 & 0.999998079 & 0.999997771 \\
\hline 1 & 5.746231847961203 & 1.001536038280316 & 1.001536023270078 \\
\hline 2 & 5.746231833605774 & 1.000033093486984 & 1.000033093488725 \\
\hline 3 & 5.746231833805267 & 1.000000000729813 & 1.000000000729813 \\
\hline 4 & 5.746231833809865 & 1.000000000000009 & 1.000000000000002 \\
\hline 6 & 5.746231833809865 & 1.000000000000000 & 1.000000000000000 \\
\hline D & 5.7461471 & 0.999998012 & 0.999997719 \\
\hline 1 & 5.746157555822260 & 1.000863731844646 & 1.000863745222875 \\
\hline $\boldsymbol{Z}$ & 5.746157545424549 & 1.000016916083231 & 1.000016916084818 \\
\hline 3 & 5.746157545577390 & 1.000000000495525 & 1.000000000495525 \\
\hline 4 & 5.746157545580572 & 1.000000000000011 & 1.000000000000011 \\
\hline 5 & 5.746157545580572 & 1.000000000000000 & 1.000000000000000 \\
\hline
\end{tabular}

The singular values displayed here are the largest ones of $W_{11}^{+}$and happen to be the closest. The matrix has a condition nuibiber of $10^{\circ}$, as a result, each iteration makes an improvemeni of approximately three digits. Note the contrast to the previous case where the matrix was well conditioned and each iteration gained a full seven digits.

\section{MULTIPIS SINGUIAR VALURS}

We are interested in improving ringe than one singular value at a time. We are motivated to do so since the approach for improving one singular value breaks down when there are multiple singular values with close numerical values. For simplicity we will restrict the discussion initially to two singular values $\sigma_{1}$ and $\sigma_{2}$ and the corresponding vectors $u_{1}, v_{1}$ and $u_{2}, v_{2}$.

We know that the two-space in which $u_{1}$ and $u_{2}$ and $v_{1}$ and $v_{2}$ lie is numerical well determined. Hence we have

$$
\begin{aligned}
& A\left(\nu_{1}+y_{1}\right)=\left(\sigma_{1}+\mu_{11}\right)\left(u_{1}+z_{1}\right)+\mu_{21}\left(u_{2}+z_{2}\right) \\
& A\left(\nu_{2}+y_{z}\right)=\mu_{15}\left(u_{1}+z_{1}\right)+\left(\sigma_{2}+\mu_{22}\right)\left(u_{2}+z_{2}\right)
\end{aligned}
$$




$$
\begin{aligned}
& A^{T}\left(u_{1}+z_{1}\right)=\left(\sigma_{1}+\mu_{11}\right)\left(v_{1}+y_{1}\right)+\mu_{21}\left(v_{2}+y_{2}\right) \\
& A^{T}\left(u_{2}+z_{2}\right)=\mu_{12}\left(v_{1}+y_{1}\right)+\left(\sigma_{2}+\mu_{22}\right)\left(v_{2}+y_{2}\right)
\end{aligned}
$$

where the corrections $y_{i}, z_{i}$, and $\mu_{i j}$ are expected to be small.

From above we form

$$
\begin{aligned}
& A\left(v_{1}+y_{1} \mid v_{2}+y_{2}\right)=\left(u_{1}+z_{1} \mid u_{2}+z_{2}\right)\left(\begin{array}{cc}
\sigma_{1}+\mu_{11} & \mu_{12} \\
\mu_{21} & \sigma_{2}+\mu_{22}
\end{array}\right) \\
& A^{T}\left(u_{1}+z_{1} \mid u_{2}+z_{2}\right)=\left(v_{1}+y_{1} \mid v_{2}+y_{2}\right)\left(\begin{array}{cc}
\sigma_{1}+\mu_{11} & \mu_{12} \\
\mu_{21} & \sigma_{2}+\mu_{22}
\end{array}\right)
\end{aligned}
$$

We will require in addition that, the orthogonality conditions

$$
\left(v_{i}+y_{i}\right)^{r}\left(v_{j}+y_{j}\right)= \begin{cases}0 & \text { if } i=j \\ 1 & \text { if } i \neq j\end{cases}
$$

and

$$
\left(u_{i}+z_{i}\right)^{T}\left(u_{j}+z_{j}\right)=\left\{\begin{array}{l}
0 \text { if } i=j \\
1 \text { if } i \neq j
\end{array}\right.
$$

be satisfied. For simplicity we will assume that the $2 \times 2$ matrix of Equation (6.2) which contains the correction to the singular values is symmetric, therefore, $\mu_{12}=\mu_{21}$. In order to produce the improved singular values this $2 \times 2$ matrix must be diagonalized.

Equation (6.2) together with Equation (6.3) give rise to $2 n+2 m+6$ equations in $2 n+2 m+6$ unknowns. This matrix equation has the form

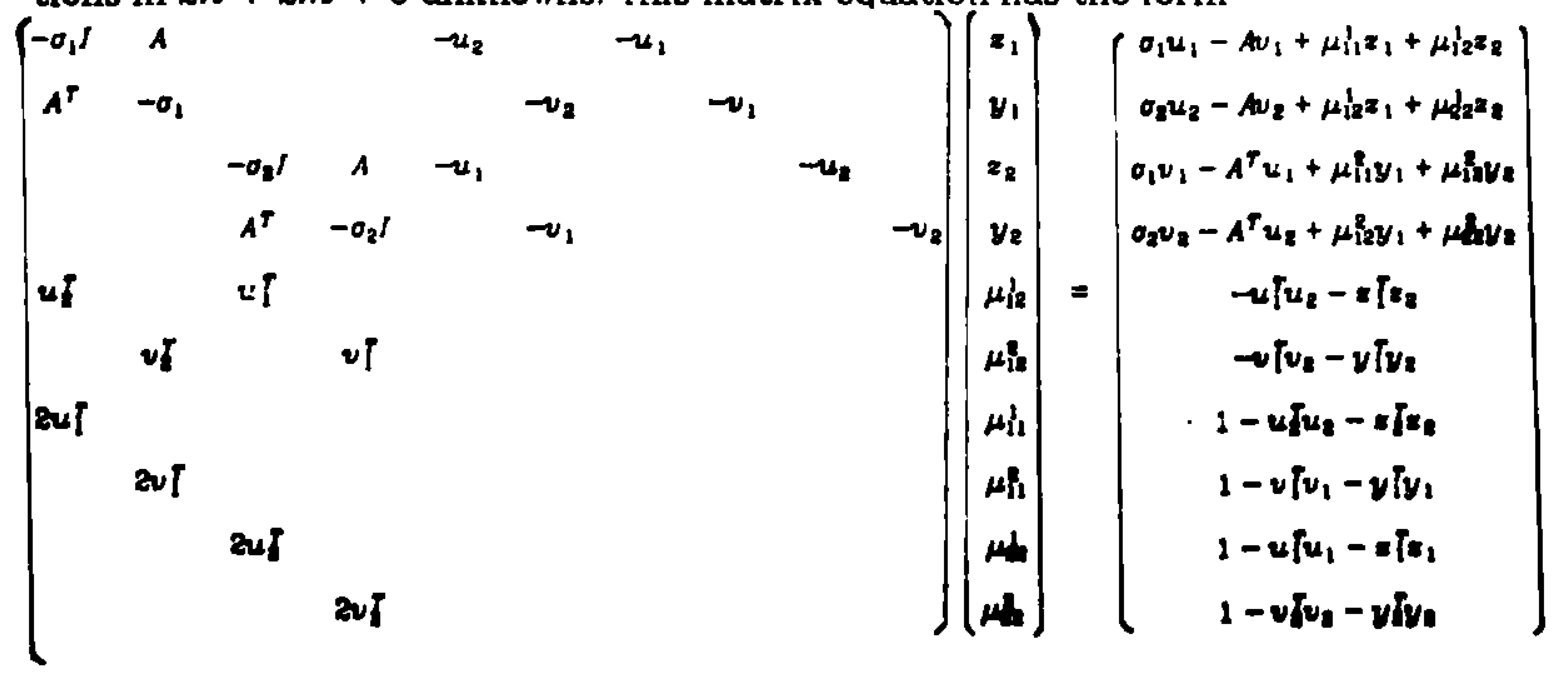


As in the case of a sirgle singular value, if one has access to the matrix factorization then the matrix problem can easily be solved.

In general, if we extend this procedure to handle $k$ close singular values we have,

$$
A\left[v_{1}+y_{1}, \ldots, v_{k}+y_{k}\right]=\left[u_{1}+z_{1}, \ldots, u_{k}+z_{k}\right]\left[\operatorname{diag}\left(\sigma_{1}\right)+M\right]
$$

and

$$
A^{T}\left[u_{1}+z_{1} \ldots, u_{k}+z_{k}\right]=\left[v_{1}+y_{1} \ldots, v_{k}+y_{k}\right]\left[\operatorname{diag}\left(\sigma_{i}\right)+M\right]
$$

where $m_{i j}=\mu_{i j}$ and $M=M^{T}$ and it is expected that $y_{i}, z_{i}$, and $\mu_{i j}$ will be small. These equations together with Equation (6.3) lead to a system of equations of order $k(m+n)+k(k+1)$ and an eigenvalue problem of order $k$.

\section{RATHRANCES}

[1] J. Dongarra, C.B. Moler and J.H. Wilkinson, Improving the Accuracy of Computed Eigenvalues and Eigenvectors, SIAM J. Numerical Analysis, to appear (Argonne National Laboratory Report, AVL-B1-43, July 1981).

[2] G.H. Golub and C. Reinsch, Singular Value Decomposition and Least Squares Solutions, Numer. Math. 14, 403-420 (1970).

[3] J H. Wilkinson. The Algebraic Eigenvalue Problem, Oxford University Press, London (1965). 